\title{
WIND TURBINE ROTOR ACCELERATION: IDENTIFICATION USING GAUSSIAN REGRESSION
}

\author{
W. E. Leithead \\ Dept of Electronic and Electrical Engineering, University of Strathclyde, Glasgow, U.K. \\ and \\ Hamilton Institute, National University of Ireland, Maynooth, Co. Kildare, Ireland \\ e-mail: w.leithead@eee.strath.ac.uk \\ Yunong Zhang, Kian Seng Neo \\ Hamilton Institute, National University of Ireland, Maynooth, Co. Kildare, Ireland \\ e-mail:ynzhang@ieee.org.,kian.neo@.strath.ac.uk
}

Keywords: Data analysis, Gaussian regression, independent processes, fast algorithms

Abstract: Gaussian processes prior model methods for data analysis are applied to wind turbine time series data to identify both rotor speed and rotor acceleration from a poor measurement of rotor speed. In so doing, two issues are addressed. Firstly, the rotor speed is extracted from a combined rotor speed and generator speed measurement. A novel adaptation of Gaussian process regression based on two independent processes rather than a single process is presented. Secondly, efficient algorithms for the manipulation of large matrices are required. The Toeplitz nature of the matrices is exploited to derive novel fast algorithms for the Gaussian process methodology that are memory efficient.

\section{INTRODUCTION}

Following some initial publications in the late 1990s (e.g., Rasmussen (1996), Gibbs (1997), Mackay (1998), Williams and Barber (1998)), interest has grown quickly into the application of Gaussian process prior models to data analysis; e.g. Williams (1999), Gibbs and Mackay (2000), Sambu et al (2000), Toshioka and Ishii (2001), Leith et al (2002), Shi et al (2003), Solak et al (2003), Leithead et al (2003a), Leithead et al (2003b). In this paper, these methods are applied to wind turbine time series data, specifically, site measurements of the rotor speed for a commercial 1MW machine. However, the measurement is some unknown combination of the rotor speed and the generator speed (scaled by the gearbox ratio) (Leithead et al, 2003b). Furthermore, the data is corrupted by significant measurement noise. The objective of the data analysis is to extract from the data both the rotor speed and the rotor acceleration, an initial yet important part of identifying the aerodynamics and drive-train dynamics of variable speed wind turbines
(Leithead et al, 2003b). Previously, only traditional filtering methods have been employed (Leithead et $a l, 2003 b)$.

To successfully identify the wind turbine rotor speed and acceleration using Gaussian process prior models, two particular issues must be addressed. Firstly, since the measurement is a combination of rotor speed and generator speed, only that contribution to the measurement due to the rotor speed must be extracted. Secondly, since analysis using Gaussian process prior models involves the inversion and multiplication of $\mathrm{N}$-dimensional square matrices where $\mathrm{N}$ is the number of data measurements $(24,000$ in this case), the matrix manipulations must be efficient. In this paper, novel adaptations of the Gaussian process data analysis methodology to meet these two issues are presented (the first in section 3 and the second in section 4) and successfully applied to the wind turbine data (in section 5). 


\section{GAUSSIAN PROCESS PRIOR MODELS}

Gaussian process prior models and their application to data analysis is reviewed in theis section.

Consider a smooth scalar function $\mathrm{f}($.) dependent on the explanatory variable, $\mathbf{z} \in \mathrm{D} \subseteq \mathfrak{R}^{\mathrm{p}}$. Suppose $\mathrm{N}$ measurements, $\left\{\left(\mathbf{z}_{\mathrm{i}}, \mathrm{y}_{\mathrm{i}}\right)\right\}_{\mathrm{i}=1}^{\mathrm{N}}$, of the value of the function with additive Gaussian white measurement noise, i.e. $\mathrm{y}_{\mathrm{i}}=\mathrm{f}\left(\mathbf{z}_{\mathrm{i}}\right)+\mathrm{n}_{\mathrm{i}}$, are available and denote them by $M$. It is of interest here to use this data to learn the mapping $\mathrm{f}(\mathbf{z})$ or, more precisely, to determine a probabilistic description of $\mathrm{f}(\mathbf{z})$ on the domain, D, containing the data. Note that this is a regression formulation and it is assumed the explanatory variable, $\mathbf{z}$, is noise free. The probabilistic description of the function, $f(\mathbf{z})$, adopted is the stochastic process, $f_{z}$, with the $E\left[f_{\mathbf{z}}\right]$, as $\mathbf{z}$ varies, interpreted to be a fit to $f(z)$. By necessity, to define the stochastic process, $f_{z}$, the probability distributions of $f_{z}$ for every choice of value of $\mathbf{z} \in D$ are required together with the joint probability distributions of $f_{z_{i}}$ for every choice of finite sample,

$\left\{\mathbf{z}_{1}, \ldots, \mathbf{z}_{\mathrm{k}}\right\}$, from $\mathrm{D}$, for all $\mathrm{k}>1$. Given the joint probability distribution for $\mathrm{f}_{\mathrm{z}_{\mathrm{i}}}, \mathrm{i}=1 . . \mathrm{N}$, and the joint probability distribution for $n_{i}, i=1 . . N$, the joint probability distribution for $\mathrm{y}_{\mathrm{i}}, \mathrm{i}=1 . . \mathrm{N}$, is readily obtained since the measurement noise, $\mathrm{n}_{\mathrm{i}}$, and the $f\left(\mathbf{z}_{\mathrm{i}}\right)$ (and so the $\mathrm{f}_{\mathbf{z}_{\mathrm{i}}}$ ) are statistically independent. $\mathrm{M}$ is a single event belonging to the joint probability distribution for $\mathrm{y}_{\mathrm{i}}, \mathrm{i}=1$..N.

In the Bayesian probability context, the prior belief is placed directly on the probability distributions describing $f_{\mathbf{z}}$ which are then conditioned on the information, $\mathrm{M}$, to determine the posterior probability distributions. In particular, in the Gaussian process prior model, it is assumed that the prior probability distributions for the $f_{\mathbf{z}}$ are all Gaussian with zero mean (in the absence of any evidence the value of $f(z)$ is as likely to be positive as negative). To complete the statistical description, requires only a definition of the covariance function $\mathrm{C}_{\mathrm{f}}\left(\mathbf{z}_{\mathrm{i}}, \mathbf{z}_{\mathrm{j}}\right)=\mathrm{E}\left[\mathrm{f}_{\mathbf{z}_{\mathrm{i}}}, \mathrm{f}_{\mathbf{z}_{\mathrm{j}}}\right]$, for all $\mathbf{z}_{\mathrm{i}}$ and $\mathbf{z}_{\mathrm{j}}$. The resulting posterior probability distributions are also Gaussian. This model is used to carry out inference as follows.

Clearly $p\left(f_{z} \mid M\right)=p\left(f_{z}, M\right) / p(M)$ where $p(M)$ acts as a normalising constant. Hence, with the Gaussian prior assumption,

$$
p\left(f_{\mathbf{z}} \mid M\right) \propto \exp \left[-\frac{1}{2}\left[\begin{array}{ll}
f_{\mathbf{z}} & \mathbf{Y}^{\mathrm{T}}
\end{array}\right]\left[\begin{array}{cc}
\Lambda_{11} & \Lambda_{21}^{\mathrm{T}} \\
\Lambda_{21} & \Lambda_{22}
\end{array}\right]^{-1}\left[\begin{array}{c}
f_{\mathbf{z}} \\
\mathbf{Y}
\end{array}\right]\right]
$$

where $\mathbf{Y}=\left[y_{1}, \cdots y_{N}\right]^{T}, \Lambda_{11}$ is $\mathrm{E}\left[\mathrm{f}_{\mathbf{z}}, \mathrm{f}_{\mathbf{z}}\right]$, the $\mathrm{ij}^{\text {th }}$ element of the covariance matrix $\Lambda_{22}$ is $E\left[y_{i}, y_{j}\right]$ and the $\mathrm{i}^{\text {th }}$ element of vector $\Lambda_{21}$ is $\mathrm{E}\left[\mathrm{y}_{\mathrm{i}}, \mathrm{f}_{\mathrm{z}}\right]$. Both $\Lambda_{11}$ and $\Lambda_{21}$ depend on $\mathbf{z}$. Applying the partitioned matrix inversion lemma, it follows that

$$
p\left(f_{\mathbf{z}} \mid M\right) \propto \exp \left[-\frac{1}{2}\left(f_{\mathbf{z}}-\hat{f}_{\mathbf{z}}\right) \Lambda_{\mathbf{z}}^{-1}\left(f_{\mathbf{z}}-\hat{f}_{\mathbf{z}}\right)\right]
$$

with $\hat{\mathrm{f}}_{\mathbf{z}}=\Lambda_{21}^{\mathrm{T}} \Lambda_{22}^{-1} \mathbf{Y}, \Lambda_{\mathbf{z}}=\Lambda_{11}-\Lambda_{21}^{\mathrm{T}} \Lambda_{22}^{-1} \Lambda_{21}$. Therefore, the prediction from this model is that the most likely value of $\mathbf{f}(\mathbf{z})$ is the mean, $\hat{f}_{z}$, with variance $\Lambda_{\mathbf{z}}$. Note that $\hat{f}_{z}$ is simply a $\mathbf{z}$-dependent weighted linear combination of the measured data points, $\mathbf{Y}$, using weights $\Lambda_{21}^{\mathrm{T}} \Lambda_{22}^{-1}$. The measurement noise, $n_{i}, i=1, . . N$, is statistically independent of $f\left(z_{i}\right)$, $\mathrm{i}=1, . . \mathrm{N}$, and has covariance matrix $\mathbf{B}$. Hence, the covariances for the measurements, $\mathrm{y}_{\mathrm{i}}$, are simply

$$
E\left[y_{i}, y_{j}\right]=E\left[f_{z_{i}}, f_{z_{j}}\right]+B_{i j} ; E\left[y_{i}, f_{z}\right]=E\left[f_{z_{i}}, f_{z}\right]
$$

In addition, assume that the related stochastic process, $f_{z}^{\delta e_{i}}$, where $f_{z}^{\delta e_{i}}=\left(f_{\left(z+\delta e_{i}\right)}-f_{z}\right) / \delta$ and $\mathbf{e}_{i}$ is a unit basis vector, is well-defined in the limit as $\delta \rightarrow 0$, i.e. all the necessary probability distributions for a complete description exist. Denote the derivative stochastic process, i.e. the limiting random process, by $\mathrm{f}_{\mathbf{z}}^{\mathbf{e}_{i}}$. When the partial derivative of $\mathrm{f}(\mathbf{z})$ in the direction $\mathbf{e}_{i}$ exists, $E\left[f_{\mathbf{z}}^{\delta \mathbf{e}_{i}}\right]$ as $\mathbf{z}$ varies is interpreted as a fit to $\frac{\partial \mathrm{f}}{\partial \mathrm{z}_{\mathrm{i}}}(\mathbf{z})$. Provided the covariance $E\left[f_{z_{i}}, f_{z_{j}}\right]$ is sufficiently differentiable, it is well known (O'Hagan, 1978) that $\mathrm{f}_{\mathbf{z}}^{\mathbf{e}_{i}}$ is itself Gaussian and that

$$
\mathrm{E}\left[\mathrm{f}_{\mathbf{z}}^{\mathbf{e}_{i}}\right]=\frac{\partial}{\partial \mathrm{z}_{\mathrm{i}}} \mathrm{E}\left[\mathrm{f}_{\mathbf{z}}\right]
$$

where $z_{i}$ denotes the $i^{\text {th }}$ element of $\mathbf{z}$; that is, the expected value of the derivative stochastic process is just the derivative of the expected value of the stochastic process. Furthermore,

$$
\begin{aligned}
& E\left[\mathrm{f}_{\mathbf{z}_{0}}^{\mathbf{e}_{\mathrm{i}}}, \mathrm{f}_{\mathbf{z}_{1}}^{\mathbf{e}_{\mathrm{j}}}\right]=\nabla_{\mathrm{i}}^{1} \nabla_{\mathrm{j}}^{2} \mathrm{E}\left[\mathrm{f}_{\mathbf{z}_{0}}, \mathrm{f}_{\mathbf{z}_{1}}\right] \\
& \mathrm{E}\left[\mathrm{f}_{\mathbf{z}_{0}}^{\mathbf{e}_{\mathrm{i}}}, \mathrm{f}_{\mathbf{z}_{1}}\right]=\nabla_{\mathrm{i}}^{1} \mathrm{E}\left[\mathrm{f}_{\mathbf{z}_{0}}, \mathrm{f}_{\mathbf{z}_{1}}\right]
\end{aligned}
$$

where $\nabla_{\mathrm{i}}^{1} \mathrm{Q}\left(\mathbf{z}_{0}, \mathbf{z}_{1}\right)$ denotes the partial derivative of $\mathrm{Q}\left(\mathbf{z}_{\mathrm{o}}, \mathbf{z}_{1}\right)$ with respect to the $\mathrm{i}^{\text {th }}$ element of its first argument, etc. 
The prior covariance function is generally dependent on a few hyperparameters, $\theta$. To obtain a model given the data, $\mathrm{M}$, the hyperparameters are adapted to maximise the likelihood, $\mathrm{p}(\mathrm{M} \mid \theta)$, or equivalently to minimize the negative log likelihood, $L(\theta)$, where

$$
L(\theta)=\frac{1}{2} \log \operatorname{det} C(\theta)+\frac{1}{2} \mathbf{Y}^{T} C(\theta)^{-1} \mathbf{Y}
$$

with $C(\theta)=\Lambda_{22}$, the covariance matrix of the measurements.

\section{MODELS WITH TWO GAUSSIAN PROCESSES}

Suppose that the measurements are not of a single function but of the sum of two functions with different characteristics; that is, the measured values are $\mathrm{y}_{\mathrm{i}}=\mathrm{f}\left(\mathbf{z}_{\mathrm{i}}\right)+\mathrm{g}\left(\mathbf{z}_{\mathrm{i}}\right)+\mathrm{n}_{\mathrm{i}}$. Now, it is of interest to use the data to learn the mappings, $\mathrm{f}(\mathbf{z})$ and $\mathrm{g}(\mathbf{z})$, or, more precisely, to determine a probabilistic description for them. The probabilistic description by means of a single stochastic process, discussed in the previous section, is no longer adequate. Instead, a novel probabilistic description in terms of the sum of two independent stochastic processes, $f_{z}$ and $g_{z}$, is proposed below.

Since $f_{\mathbf{z}}$ and $g_{z}$ are independent, $E\left[\mathbf{F G}^{\mathrm{T}}\right]=0$ where $\mathbf{F}=\left[\mathrm{f}_{\mathbf{z}_{1}}, \cdots \mathrm{f}_{\mathbf{z}_{\mathrm{N}}}\right]^{T}$ and $\mathbf{G}=\left[\mathrm{g}_{\mathbf{z}_{1}}, \cdots \mathrm{g}_{\mathbf{z}_{\mathrm{N}}}\right]^{T}$. Let the covariance functions for $f_{z}$ and $g_{z}$ be $C_{f}\left(\mathbf{z}_{i}, \mathbf{z}_{j}\right)$ and $\mathrm{C}_{\mathrm{g}}\left(\mathbf{z}_{\mathrm{i}}, \mathbf{z}_{\mathrm{j}}\right)$, respectively. Note that this is a different model from one using a single stochastic process with covariance function, $\left(\mathrm{C}_{\mathrm{f}}+\mathrm{C}_{\mathrm{g}}\right)$. It follows that

$$
\begin{aligned}
& \Lambda=\mathrm{E}\left[\left[\begin{array}{c}
\mathbf{F} \\
\mathbf{G} \\
\mathbf{Y}
\end{array}\right]\left[\begin{array}{lll}
\mathbf{F}^{T} & \mathbf{G}^{T} & \mathbf{Y}^{T}
\end{array}\right]=\left[\begin{array}{ccc}
\Lambda_{\mathrm{FF}} & 0 & \Lambda_{\mathrm{FF}} \\
0 & \Lambda_{\mathbf{G G}} & \Lambda_{\mathbf{G G}} \\
\Lambda_{\mathrm{FF}} & \Lambda_{\mathbf{G G}} & \mathbf{Q}
\end{array}\right]\right. \\
& \text { with } \Lambda_{\mathbf{F F}}=\mathrm{E}\left[\mathbf{F F}^{T}\right], \quad \Lambda_{\mathbf{G G}}=\mathrm{E}\left[\mathbf{G G} \mathbf{G}^{T}\right] \quad \text { and } \\
& \mathbf{Q}=\mathbf{B}+\Lambda_{\mathbf{F F}}+\Lambda_{\mathbf{G G}} .
\end{aligned}
$$

The prior joint probability distribution for $\mathbf{F}, \mathbf{G}$ and $\mathbf{Y}$ is Gaussian with mean zero and covariance matrix $\Lambda$. The requirement is to obtain the posterior probability distribution for $\mathbf{F}$ and $\mathbf{G}$ conditioned on the data set, M, subject to the condition that they remain independent. Of course, the posterior probability distribution remains Gaussian. The mean and covariance matrix for the posterior is provided by the following theorem (Leithead et al, 2005).
Theorem 1: Given that the prior joint probability distribution for $\mathbf{F}, \mathbf{G}$ and $\mathbf{Y}$ is Gaussian with mean zero and covariance matrix $\Lambda$, the posterior joint probability distribution for $\left[\mathbf{F}^{\mathbf{T}}, \mathbf{G}^{\mathbf{T}}\right]^{\mathrm{T}}$ conditioned on the $\mathrm{M}$, subject to the condition that they remain independent, is Gaussian with

$$
\begin{aligned}
& \text { mean }=\left[\begin{array}{c}
\Lambda_{\mathbf{F F}} \mathbf{Q}_{\mathbf{F}}^{-1} \mathbf{Y} \\
\mathbf{B} \mathbf{Q}_{\mathbf{F}}^{-1} \Lambda_{\mathbf{G G}} \mathbf{Q}^{-1} \mathbf{Y}
\end{array}\right] \\
& \operatorname{cov}=\left[\begin{array}{cc}
\Lambda_{\mathbf{F F}} \mathbf{Q}_{\mathbf{F}}^{-1} \mathbf{B} & 0 \\
0 & \mathbf{B} \mathbf{Q}_{\mathbf{F}}^{-1} \Lambda_{\mathbf{G G}} \mathbf{Q}^{-1} \mathbf{B}
\end{array}\right]
\end{aligned}
$$

where $\mathbf{Q}_{\mathbf{F}}=\mathbf{B}+\Lambda_{\mathbf{F F}}$.

Proof. Omitted due to space limitations.

Note, since $f_{z}$ and $g_{z}$ remain independent when conditioned on $\mathrm{M}$, the prediction and covariance for $\left(f_{\mathbf{z}}+g_{\mathbf{z}}\right)$ are simply the sum of the individual predictions and covariance values.

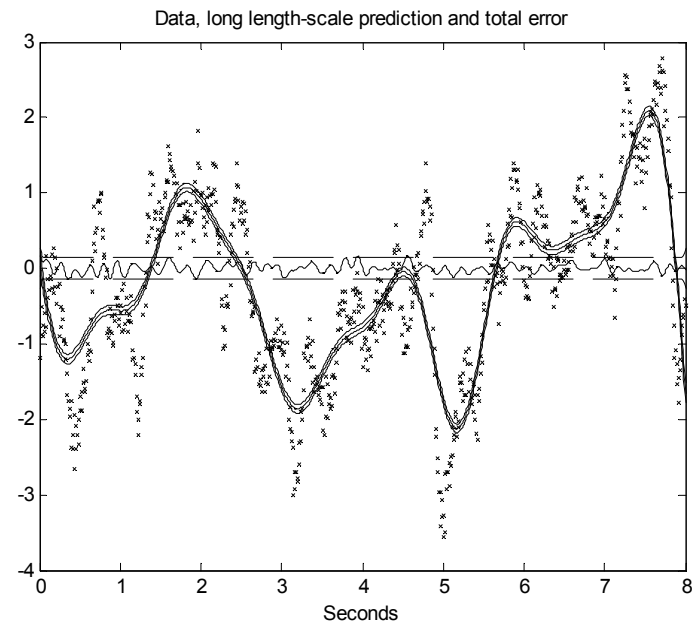

Figure 1: Data values $(* * *)$, long length-scale component with confidence intervals $(-)$ and total error and confidence interval $(==)$.

Example: A commonly used prior covariance function for a Gaussian process with scalar explanatory variable is

$$
a \exp \left[-\frac{1}{2} d\left(\mathrm{z}_{i}-\mathrm{z}_{j}\right)^{2}\right]
$$

It ensures that measurements associated with nearby values of the explanatory variable should have higher correlation than more widely separated values of the explanatory variable; $a$ is related to the overall mean amplitude and $d$ inversely related to the length-scale of the Gaussian process.

Let the covariance function for $f_{\mathbf{z}}$ be (9) with $\mathrm{a}=1.8$ and $\mathrm{d}=2.5$, and the covariance function for $\mathrm{g}_{\mathrm{z}}$ be (9) with $a=0.95$ and $d=120$; that is, $f_{\mathbf{z}}$ has a long 
length-scale and $g_{z}$ a short length-scale. In addition, let the measurement noise be Gaussian white noise with variance $b=0.04$, i.e. $B_{i j}=b \delta_{i j}$, where $\delta_{i j}$ is the Kronecker delta. A set of 800 measurements at constant interval, 0.01 , for $\mathrm{y}_{\mathrm{i}}=\mathrm{f}\left(\mathbf{z}_{\mathrm{i}}\right)+\mathrm{g}\left(\mathbf{z}_{\mathrm{i}}\right)+\mathrm{n}_{\mathrm{i}}$, with the $\mathrm{f}\left(\mathbf{z}_{\mathrm{i}}\right)$ and $\mathrm{g}\left(\mathbf{z}_{\mathrm{i}}\right)$ the sample values for the stochastic processes $f_{z}$ and $g_{z}$, respectively, is shown in figure 1.

A prediction for the long and short length-scale components is obtained using (8); that is, the conditioning on the data is chosen such that as much of the data as possible is explained by the long length-scale component. The long length-scale component with its confidence interval ( \pm 2 standard deviations) is shown in Figure 1 and the short length-scale component with its confidence interval is shown in Figure 2. The prediction error for $\left(\mathrm{f}_{\mathbf{z}}+\mathrm{g}_{\mathrm{z}}\right)$ with its confidence interval is also depicted in Figure 2.

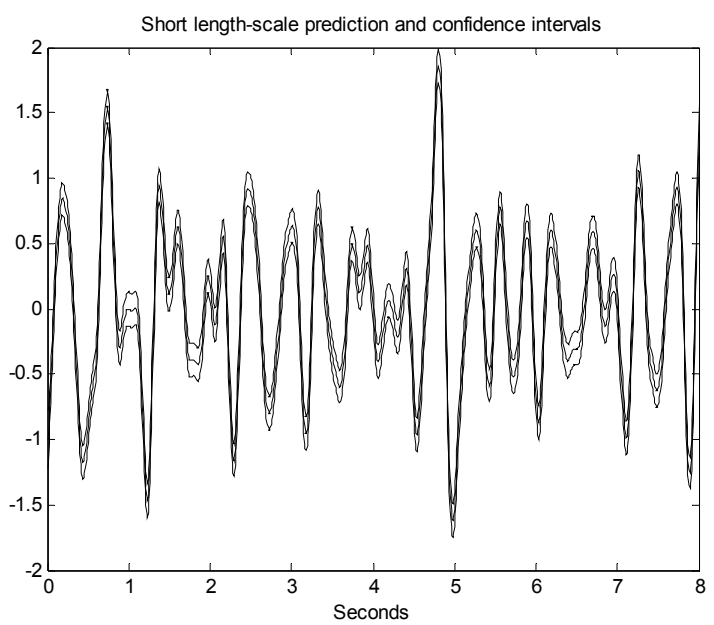

Figure 2: Short length-scale component with confidence intervals.

In Section 5, Theorem 1 is applied to the wind turbine measurement data to extract the contribution due to the rotor speed and, together with (4) and (5), to identify the rotor acceleration.

\section{TOEPLITZ-BASED EFFICIENCY IMPROVEMENT}

In section 3, a novel adaptation of the Gaussian regression methodology to support the extraction of separate components from data is presented. However, before that procedure can be applied to large data sets, fast and memory efficient algorithms are required. That requirement is addressed in this section.

As the log likelihood, (6), is in general nonlinear and multimodal, efficient optimisation routines usually need gradient information,

$$
\frac{\partial L}{\partial \theta_{i}}=\frac{1}{2} \operatorname{tr}\left(C^{-1} \frac{\partial C}{\partial \theta_{i}}\right)-\frac{1}{2} \mathbf{Y}^{T} C^{-1} \frac{\partial C}{\partial \theta_{i}} C^{-1} \mathbf{Y}
$$

where $\theta_{i}$ denotes the $i$-th hyperparameter and $\operatorname{tr}(\cdot)$ the trace operation of a matrix. Let us denote $\partial C / \partial \theta_{i}$ hereafter by $P$ for notational convenience. Clearly, in general, the number of operations in solving for $\log \operatorname{det} C$ and $C^{-1}$ is of $O\left(N^{3}\right)$, whilst the memory space to store $C, C^{-1}$ and $P$ is $O\left(N^{2}\right)$ (see Table 1 for specific values). For large data sets, fast algorithms, that require less memory allocation, are required for the basic matrix manipulations, $\log \operatorname{det} C, C^{-1} y$ and $\operatorname{tr}\left(C^{-1} P\right)$, when tuning the Gaussian process prior model hyperparameters.

Now consider a time series with fixed interval between the measurements. The explanatory variable, $\mathbf{z}$, in the Gaussian process prior model is a scalar, the time $t$. When the covariance function depends on the difference in the explanatory variable, as is almost always the case, the covariance matrix $C(\theta)$ and its derivative matrices $P$ are Toeplitz; that is,

$$
\begin{aligned}
C & =\left[\begin{array}{cccc}
c_{0} & c_{1} & \cdots & c_{N-1} \\
c_{1} & c_{0} & \cdots & c_{N-2} \\
\vdots & \vdots & \ddots & \vdots \\
c_{N-1} & c_{N-2} & \cdots & c_{0}
\end{array}\right]=\operatorname{Toeplitz}(c) \\
c & =\left[\begin{array}{llll}
c_{0} & c_{1} & \cdots & c_{N-1}
\end{array}\right]
\end{aligned}
$$

Furthermore, $C(\theta)$ is symmetric and positive definite.

Here, the Toeplitz nature of $C(\theta)$ is exploited to derive novel fast algorithms for the Gaussian process methodology that require less memory allocation. It is well-known that positive-definite Toeplitz matrices can be elegantly manipulated in $O\left(N^{2}\right)$ operations. For example, Trench's algorithm inverts $C$ with $13 N^{2} / 4$ operations, and Levinson's algorithm solves for $C^{-1} y$ with $4 N^{2}$ operations (Golub \& Van Loan, 1996). However, direct application of these algorithms to Gaussian process regression may fail even with medium-scale datasets due to lack of memory space, see Table 1. For example, on a Pentium-IV 3GHz 512MB-RAM PC, 
a MATLAB-JAVA algorithm usually fails around $N=7000$ because storing $C^{-1}$ almost uses up the available system memory. The solution is to adapt the fast algorithms to use only vector-level storage. From Table 1 that approach is theoretically able to handle very large datasets, such as 1-million data points, in terms of memory requirements.

Table 1: Memory/storage requirement in double precision.

\begin{tabular}{ccc}
\hline \hline$N$ & Matrix & Vector \\
\hline 1000 & $7.7 \mathrm{MB}$ & $7.6 \mathrm{~KB}$ \\
7000 & $373.9 \mathrm{MB}$ & $53.4 \mathrm{~KB}$ \\
15000 & $1716.6 \mathrm{MB}$ & $114.4 \mathrm{~KB}$ \\
20000 & & $152.6 \mathrm{~KB}$ \\
30000 & & $228.9 \mathrm{~KB}$ \\
50000 & & $381.5 \mathrm{~KB}$ \\
100000 & & $762.9 \mathrm{~KB}$ \\
1000000 & & $7.629 \mathrm{MB}$ \\
\hline \hline
\end{tabular}

Two versions of fast Toeplitz-computation algorithms are discussed and compared below; namely, a full-matrix version and a vector-storage version.

\subsection{Full-Matrix Toeplitz Computation}

The simplest way of applying Toeplitz computation to Gaussian process regression is to compute $C^{-1}$ directly as the basis for the other matrix manipulations. Specifically, Trench's algorithm of $O\left(N^{2}\right)$ operations can be readily modified to obtain $C^{-1}$ whilst simultaneously determining $\log \operatorname{det} C$ as the logarithm sum of the reflection coefficients. Then, given $C^{-1}$, the computation $\operatorname{tr}\left(C^{-1} P\right)=\sum_{i} \sum_{j} \bar{c}_{i j} p_{i j} \quad$ is easily performed in $O\left(N^{2}\right)$ operations, where $\bar{c}_{i j}$ and $p_{i j}$ are the $i j$-th elements of $C^{-1}$ and $P$, respectively.

Table 2: Accuracy and speedup of Toeplitz computation.

\begin{tabular}{c|c|c|c}
\hline & $N=1000$ & $N=2000$ & $N=3000$ \\
\hline $\begin{array}{c}\text { Accuracy on } \\
\log \operatorname{det} C\end{array}$ & $4.1 \times 10^{-15}$ & $3.4 \times 10^{-15}$ & $3.5 \times 10^{-15}$ \\
$C^{-1} y$ & $1.3 \times 10^{-12}$ & $7.0 \times 10^{-13}$ & $2.2 \times 10^{-12}$ \\
$\operatorname{tr}\left(C^{-1} P\right)$ & $8.1 \times 10^{-14}$ & $1.7 \times 10^{-13}$ & $5.5 \times 10^{-14}$ \\
\hline Speed up & 70.45 & 91.64 & 90.84 \\
\hline \multicolumn{4}{r}{}
\end{tabular}

Note that Trench's algorithm uses Durbin's algorithm to solve Yule-Walker equations (Golub \& Van Loan, 1996). These two algorithms are implemented separately; specifically, the matrix-free algorithm that generates an instrumental vector and the remaining part that generates $C^{-1}$. The former does not contain any matrix or matrix-related computation/storage, and is thus able to perform very high-dimension Toeplitz-computation. In view of this, it is also used in Section 4.2 as a part of the vector-storage version of Toeplitz-computation.

A large number of numerical experiments are performed to verify the correctness of the modified algorithms and their implementation. The covariance function is

$$
a \exp \left[-\frac{1}{2} d\left(\mathrm{z}_{i}-\mathrm{z}_{j}\right)^{2}\right]+b \delta_{i j}
$$

with random hyperparameters, $a \in(0,3)$, $d \in(0,0.05), b \in(0,0.3)$. The numerical stability, accuracy and speed-up of the algorithms are compared to the standard MATLAB matrixinversion routines, see Table 2 where the mean of the relative errors and speed-up ratios are shown. Each test is based on 100 random covariance matrices. Trench's algorithm is sufficiently stable for the Gaussian process context in the sense that it can work well for $d / a \geq 10^{-11}$, though it is slightly less stable than the MATLAB INV routine (the latter can work well for $d / a \geq 10^{-15}$ ).

\subsection{Vector-Storage Toeplitz Computation}

As discussed above, the full-matrix Toeplitz computation works well for medium-scale regression tasks with a speed-up of around 100 . However, the matrix-level memory allocation is still an issue for large datasets with $N$ greater than 7,000 such as the wind-turbine data. It follows from Table 1 , that if possible, a specialized vector-level storage version of the algorithms is attractive for specific computation task, such as Gaussian regression for time series.

The modified matrix-free Durbin's algorithm, see above, is used to compute $\log \operatorname{det} C$ and Levinson's algorithm to compute $C^{-1} y$. The remaining manipulation, namely $\operatorname{tr}\left(C^{-1} P\right)$, is obtained with the aid of the following theorem.

Theorem 2. $\operatorname{tr}\left(C^{-1} P\right)$ can be computed as 


$$
\operatorname{tr}\left(C^{-1} P\right)=\varphi_{1} p_{1}+2 \sum_{i=2}^{N} \varphi_{i} p_{i}
$$

where $P$ is Toeplitz with representative vector $p=\left[p_{1}, p_{2}, \cdots, p_{N}\right]$ and $\varphi_{i}$ denotes the summation of the elements in the $i$ th diagonal of $C^{-1}$.

Proof. Omitted due to space limitation.

Table 3: Accuracy and run-time of Toeplitz computation.

\begin{tabular}{c|c|c}
\hline & $\begin{array}{c}\text { Accuracy in the form } \\
\text { of mean (std) }\end{array}$ & $\begin{array}{c}\text { Time } \\
\text { (seconds) }\end{array}$ \\
\hline$N=10000$ & $1.4 \times 10^{-13}\left(2.0 \times 10^{-13}\right)$ & $29.1(22.5)$ \\
\hline$N=20000$ & $2.0 \times 10^{-13}\left(3.7 \times 10^{-13}\right)$ & $\begin{array}{c}264.7 \\
(154.2)\end{array}$ \\
\hline$N=30000$ & $1.4 \times 10^{-13}\left(2.1 \times 10^{-13}\right)$ & $\begin{array}{c}730.8 \\
(393.3)\end{array}$ \\
& & $\begin{array}{c}1555.3 \\
(784.8)\end{array}$ \\
\hline$N=40000$ & $2.5 \times 10^{-13}\left(5.6 \times 10^{-13}\right)$ & 2497.1 \\
& & $(1339.3)$ \\
\hline$N=50000$ & $2.8 \times 10^{-13}\left(1.4 \times 10^{-12}\right)$ & 3641.7 \\
& & $(1957.0)$ \\
\hline$N=60000$ & $1.4 \times 10^{-13}\left(2.2 \times 10^{-13}\right)$ & \\
& &
\end{tabular}

Before applying them to Gaussian process regression, a large number of numerical experiments are also performed for the efficient and economical vector-storage version of the Toeplitz algorithms. Random covariance matrices are generated and tested as in the previous subsection. Table 3 shows the numerical accuracy and execution time of the algorithms (the standard deviation is given in brackets). The results substantiate the efficacy of the vector-storage Toeplitz computation on large datasets.

\section{WIND TURBINE DATA}

The measurement data for the wind turbine rotor speed consist of a run of 600 seconds sampled at $40 \mathrm{~Hz}$. A typical section, from $200 \mathrm{~s}$ to 400 s, is shown in Figure 3. The data has a long length-scale component due to variations in the aerodynamic torque, caused by changes in the wind speed and the pitch angle of the rotor blades, and a short lengthscale component due to the structural and electromechanical dynamics of the machine. From Figure 3 , these two components can be clearly seen as can the poor quality of the data.



Figure 3: Rotor speed measurements from $200 s$ to $400 s$.



Figure 4: Rotor speed prediction, confidence intervals and data from $265 s$ to $275 s$.

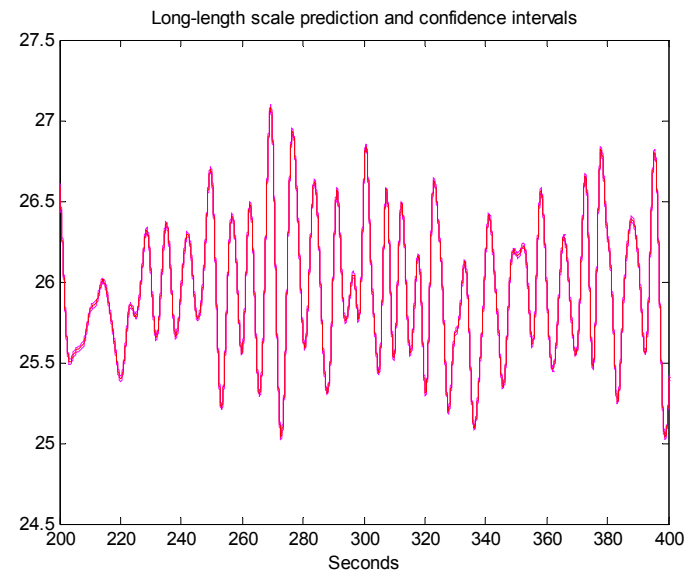

Figure 5: Rotor speed prediction with confidence intervals. 
It is required to estimate the long-length-scale component in the rotor speed. Since the structural and electro-mechanical dynamics only induce small oscillations in the measured values, a prediction of the rotor speed using (8) is appropriate with $f_{z}$ and $g_{z}$ the long and short-length components respectively. The covariance function for $\mathrm{f}_{\mathbf{z}}$ is chosen to have the form (9) with hyperparameters $a_{\mathrm{f}}$ and $d_{\mathrm{f}}$ as is the covariance function for $\mathrm{g}_{\mathrm{z}}$ with hyperparameters $a_{\mathrm{g}}$ and $d_{\mathrm{g}}$. The measurement noise is assumed to be Gaussian white noise with variance $b$. Hence, the prior covariance for the measurements, $y_{\mathrm{i}}$, at time, $t_{\mathrm{i}}$, are

$$
\begin{aligned}
\mathrm{E}\left[\mathrm{y}_{\mathrm{i}}, \mathrm{y}_{\mathrm{j}}\right] & =a_{\mathrm{f}} \exp \left[-\frac{1}{2} d_{\mathrm{f}}\left(\mathrm{t}_{\mathrm{i}}-\mathrm{t}_{\mathrm{j}}\right)^{2}\right] \\
& +a_{\mathrm{g}} \exp \left[-\frac{1}{2} d_{\mathrm{g}}\left(\mathrm{t}_{\mathrm{i}}-\mathrm{t}_{\mathrm{j}}\right)^{2}\right]+b \delta_{i j}
\end{aligned}
$$

Given the data, the hyperparameters are adapted to maximize the likelihood. Since there are 24,000 data values, it is necessary to use the vector-storage Toeplitz algorithms of Section 4.2.

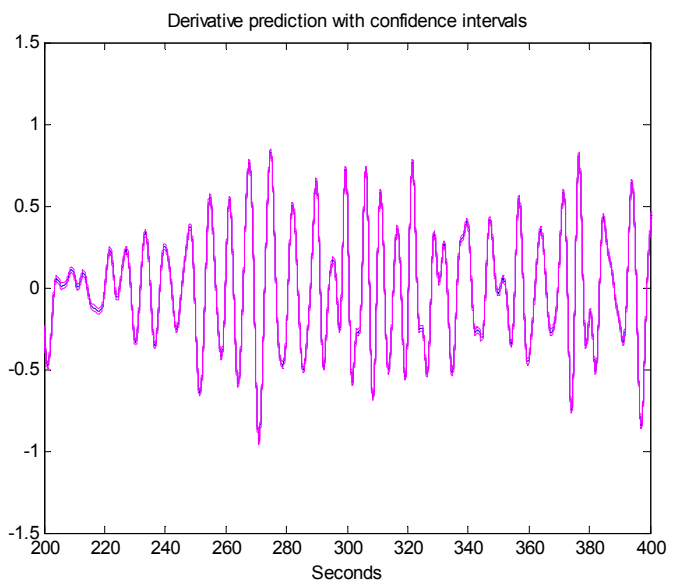

Figure 6: Derivative prediction with confidence intervals.



Figure 7: Derivative prediction with confidence intervals.
A section, from $200 s$ to $400 s$, of the prediction for the rotor speed, i.e. the long length-scale component, together with the confidence intervals is shown in Figure 5 and a typical short section, from $265 \mathrm{~s}$ to $275 s$, in Figure 4. From the latter, it can be seen that the rotor speed has been successfully extracted. However, it is not the rotor speed per se that is of interest but its derivative. A section, from $200 \mathrm{~s}$ to $400 s$, of the prediction for the derivative of the rotor speed together with the confidence intervals is shown in Figure 6 and a short section, from $265 s$ to $275 s$, in Figure 7.

\section{CONCLUSIONS}

From poor quality wind turbine rotor speed measurements, the rotor speed and acceleration are estimated within narrow confidence intervals using Gaussian process regression. To do so, two issues are addressed. Firstly, the rotor speed is extracted from a combined rotor speed and generator speed measurement. A novel adaptation of Gaussian process regression based on two independent processes rather than a single process is presented. Secondly, efficient algorithms for the manipulation of large matrices $(24,000 \times 24,000)$ are required. The Toeplitz nature of the matrices is exploited to derive novel fast algorithms for the Gaussian process methodology that are memory efficient.

\section{ACKNOWEDGEMENTS}

This work was supported by Science Foundation Ireland grant, 00/PI.1/C067, and by the EPSRC grant, GR/M76379/01.

\section{REFERENCES}

Gibbs, M. N., 1997. Bayesian Gaussian processes for regression and classification, Ph.D. thesis, Cambridge University.

Gibbs, M. N., and Mackay, D. J. C., 2000. Variational Gaussian process classifiers, IEEE Transactions on Neural Networks, Vol. 11, pp. 1458-1464.

Golub, G. H., and Van Loan, C. F. , 1996. Matrix Computations. Baltimore: Johns Hopkins University Press. 
Leith, D. J., Leithead, W. E., Solak, E., and MurraySmith, R., 2002. Divide and conquer identification using Gaussian process priors, Proceedings of the 41 st IEEE Conference on Decision and Control, Vol. 1 1, pp. 624-629.

Leithead, W. E., Solak, E., and Leith, D., 2003a. Direct identification of nonlinear structure using Gaussian process prior models, Proceedings of European Control Conference, Cambridge.

Leithead, W. E., Hardan, F., and Leith, D. J., 2003b, Identification of aerodynamics and drive-train dynamics for a variable speed wind turbine, Proceedings of European Wind Energy Conference, Madrid.

Leithead, W. E., Kian Seng Neo, Leith, D. J., 2005. Gaussian regression based on methods with two stochastic processes, to be presented, IFAC, Prague, 2005.

Mackay, D. J. C., 1998. Introduction to Gaussian processes. In Neural Networks and Machine Learning, F: Computer and Systems Sciences (Bishop, C. M., Ed.), Vol. 168, pp. 133-165, Springer: Berlin, Heidelberg.

O'Hagan, A., 1978. On curve fitting and optimal design for regression, J. Royal Stat Soc. B, 40, pp. 1-42.

Rasmussen, C. E., 1996. Evaluation of Gaussian processes and other methods for non-linear regression, Ph.D. thesis, University of Toronto.

Sambu, S., Wallat, M., Graepel, T., and Obermayer, K., 2000. Gaussian process regression: active data selection and test point rejection, Proceedings of the IEEE International Joint Conference on Neural Networks, Vol. 3, pp. 241-246.

Shi, J. Q., Murray-Smith, R., and Titterington, D. M., 2003. Bayesian regression and classification using mixtures of multiple Gaussian processes, International Journal of Adaptive Control and Signal Processing, Vol. 1 17, pp. 149-161.

Solak, E., Murray-Smith, R.,. Leithead, W. E., Leith, D., and Rasmussen, C. E., 2003. Derivative observations in Gaussian process models of dynamic systems, Advances in Neural Information Processing Systems, Vol. 15, pp. 1033-1040, MIT Press.

Williams, C. K. I., 1999. Prediction with Gaussian processes: from linear regression to linear prediction and beyond, Learning in Graphical Models (Jordan, M. I., Ed.), pp. 599-621.

Williams, C. K. I., and Barber, D., 1998. Bayesian classification with Gaussian processes, IEEE Transactions on Pattern Analysis and Machine Intelligence, Vol. 120 , pp. 1342-1351.

Yoshioka, T., Ishii, S., 2001. Fast Gaussian process regression using representative data, Proceedings of International Joint Conference on Neural Networks, Vol. 11 , pp. 132-137. 